Research Paper

\title{
Macrophage Migration Inhibitory Factor Promotes Proliferation and Neuronal Differentiation of Neural Stem/Precursor Cells through Wnt/ $\beta$-Catenin Signal Pathway
}

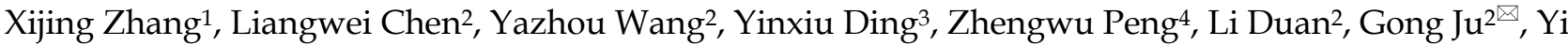 \\ Ren $^{5 凶}, X_{i}$ Wang ${ }^{2 凶}$ \\ 1. Department of Anesthesiology, Xijing Hospital, the Fourth Military Medical University, Xi' an 710032, China \\ 2. Institute of Neurosciences, the Fourth Military Medical University, Xi'an 710032, China \\ 3. Department of Human Anatomy, Ningxia Medical University, Yinchuan 750004, China \\ 4. Department of Psychosomatic Medicine, Xijing Hospital, the Fourth Military Medical University, Xi'an 710032, China \\ 5. W. M. Keck Center for Collaborative Neuroscience, Rutgers, the State University of New Jersey, NJ 08854, USA
}

$\square$ Corresponding authors: Xi Wang, Institute of Neurosciences, the Fourth Military Medical University, Xi'an 710032, China. Email: wangzh@fmmu.edu.cn; (or) Yi Ren, W. M. Keck Center for Collaborative Neuroscience, Rutgers, the State University of New Jersey, Piscataway, NJ 08854 USA. Email: ren@dls.rutgers.edu. The present address: Department of Biomedical Sciences, Florida State University College of Medicine, 1115 West Call Street, Tallahassee, FL 32306; (or) Gong Ju, Institute of Neurosciences, the Fourth Military Medical University, Xi'an 710032, China. Email: jugong@fmmu.edu.cn

( ) Ivyspring International Publisher. This is an open-access article distributed under the terms of the Creative Commons License (http://creativecommons.org/ licenses/by-nc-nd/3.0/). Reproduction is permitted for personal, noncommercial use, provided that the article is in whole, unmodified, and properly cited.

Received: 2013.07.23; Accepted: 2013.10.23; Published: 2013.11.28

\begin{abstract}
Macrophage migration inhibitory factor (MIF) is a highly conserved and evolutionarily ancient mediator with pleiotropic effects. Recent studies demonstrated that the receptors of MIF, including CD44, CXCR2, CXCR4 and CD74, are expressed in the neural stem/progenitor cells (NSPCs). The potential regulatory effect of MIF on NSPCs proliferation and neuronal differentiation, however, is largely unknown. Here, we investigated the effect of MIF on NSPC proliferation and neuronal differentiation, and further examined the signal pathway by which MIF transduced these signal effects in mouse NSPCs in vitro. The results showed that both Ki67-positive cells and neurosphere volumes were increased in a dose-dependent manner following MIF treatment. Furthermore, the expression of nuclear $\beta$-catenin was significantly stronger in MIF-stimulated groups than that in control groups. Conversely, administration of IWR-I, the inhibitor of Wnt/ $\beta$-catenin pathway, significantly inhibited the proliferative effect of MIF on NSPCs. Immunostaining and Western blot further indicated that doublecortin (DCX) and Tuj I, two neuronal markers, were evidently increased with MIF stimulation during NSPC differentiation, and there were more Tujl-positive cells migrated out from neurospheres in MIF-stimulated groups than those in control groups. During NSPC differentiation, MIF increased the activity of $\beta$-galactosidase that responds to $\mathrm{Wnt} / \beta$-catenin signaling. $\mathrm{Wntl}$ and $\beta$-catenin proteins were also up-regulated with MIF stimulation. Moreover, the expression of DCX and Tuj I was inhibited significantly by IWR-I. Taken together, the present study indicated that MIF enhances NSPC proliferation and promotes the neuronal differentiation, by activating $W n t / \beta$-catenin signal pathway. The interaction between MIF and $\mathrm{Wnt} / \beta$-catenin signal pathway may play an important role in modulating NSPC renewal and fate during brain development.
\end{abstract}

Key words: MIF; NSPC; proliferation; neuronal differentiation; Wnt/ $\beta$-catenin 


\section{Introduction}

Neural stem/progenitor cells (NSPCs) are self-renewing cells and can differentiate into multiple neural lineages during embryo development and perinatal period. These cells are essential for brain development and brain physiological functions. Therefore, a better understanding of the fine regulatory mechanism of proliferation and neuronal differentiation from NSPCs is required.

The niche where NSPCs reside is a microenvironment that maintains the self-renewal and the multipotent state of the NSPCs. The components in the niches are the source of extrinsic signals to instruct the NSPC to either self-renew or differentiate and influence the decision of NSPC's fate into neuron or glia [1]. Cytokines are proved to be one kind of the extracellular regulators in the niches and involved in determining NSPC's fate [2] and regulate the function of NSPCs. The most studied cytokines include: interleukin (IL) -1, IL-6, tumor necrosis factor (TNF) - $\alpha$, insulin-like growth factor-1, growth-regulated oncogene- $\alpha$, leukemia inhibitory factor, cardiotrophin-1, ciliary neurotrophic factor, interferon- $\gamma$, monocyte chemotactic protein-1 and macrophage inflammatory protein-1 $\alpha$ [3]. The pro-inflammatory cytokines, such as IL-1 $\beta$, IL- 6 and TNF- $\alpha$, mostly trigger astrocyte differentiation and inhibit NSPC self-renewal [2].

However, the macrophage migration inhibitory factor (MIF), one of the first cytokines identified, has yet to be clarified in the NSPC self-renewal and differentiation. MIF was initially discovered as a lymphocyte-derived protein capable of preventing the random migration of macrophages and recruiting them to inflamed sites $[4,5]$. Recently, increasing evidence indicates that MIF functions not only as its historic name, but also as a pleiotropic molecule [6]. MIF can act as an anterior pituitary hormone, a pro-inflammatory cytokine, and high activity enzyme, and is well known for its role in many inflammation-based diseases [7-9]. MIF is ubiquitously expressed in various types of tissues and cells, including the cells in the CNS $[10,11]$. In the brain, MIF transcripts and protein are found in astrocyte, microglia, neuron and NSPC [10-12]. Most importantly, MIF receptors, CD74/CD44, CXCR2 and CXCR4, were found in NSPCs cells, suggesting that MIF can maintain NSPCs through autocrine and/or paracrine mechanisms [12]. MIF was demonstrated to be a ligand of CD74 [13], and a non-cognate ligand for CXCR2 and CXCR4 [14]. CD74 can generate a complex with CD44 and act as a receptor for MIF [15]. However, the function of MIF in the CNS is largely unknown. Although a few of recent studies indicated that MIF has broad roles in regulation of neuronal degeneration-regeneration processes $[11,16-20]$ and neural stem/ progenitor cell survival and proliferation [12], the conclusion is not completely consistent yet. In our previous studies, we found that MIF was capable of promoting the proliferation of embryonic stem cells [21]. Therefore, the aim of the present study is to examine whether MIF play a similar role on NSPC proliferation and differentiation.

It is well-known that canonical Wnt signal pathway, mediated through $\beta$-catenin plays a critical role in neural development and growth. When $\beta$-catenin expression is increased in the subventricular zone (SVZ) of adult mice, the proliferation of neural precursors is increased [22]. Mice that express activated $\beta$-catenin in the cerebellar ventricular zone exhibit increased proliferation of NSPCs [23]. The $\beta$-catenin activation in neural precursors can cause an increase in neuronal number in the adult forebrain [24]. Although the role of canonical Wnt signal pathways in neurogenesis has been studied extensively, the interactions of MIF and canonical Wnt/ $\beta$-catenin signal pathway, as well as the functions of their interactions, in neurogenesis remain largely unclear.

We hypothesize that MIF signaling may play a critical role in regulating networks of NSPC proliferation and differentiation, in which MIF and Wnt/ $\beta$-catenin signaling may have an important functional interaction. Our results provide evidence that MIF promotes the proliferation and neuronal differentiation of NSPCs through Wnt/ $\beta$-catenin signal pathway.

\section{Materials and methods}

\section{Animal}

Two-day-old pups of C57BL/6J mouse and TOPGAL transgenic mice expressing $\beta$-galactosidase in the presence of the lymphoid enhancer binding factor 1 /transcription factor 3 (LEF/TCF) mediated signaling pathway and activated $\beta$-catenin (LacZ ${ }^{\text {TCF }}$ $\mathrm{Tg}$ mice) [25] were used. The expression of $\beta$-galactosidase can indicate the activation of Wnt/ $\beta$-catenin signal pathway. Pups of C57BL/6J mouse were provided by the Laboratory Animal Center of the Fourth Military Medical University. LacZ ${ }^{\text {TCF }} \mathrm{Tg}$ mice were obtained from Jackson Lab. All methods of experiments were carried out in accordance with the Guidelines for Animal Experimentation and performed in compliance with the Ethics Committee for Animal Experimentation of the Fourth Military Medical University.

\section{Cell culture}

NSPCs were cultured and differentiated as described by Ohta et al. [12]. Briefly, septum and stria- 
tum were isolated from 2-day-old pup brains. Tissues were dispersed mechanically with a pipette up and down in $1 \mathrm{ml}$ of $0.05 \%$ trypsin within 2 minutes followed by adding $1 \mathrm{ml}$ of trypsin inhibitor $(0.25 \mathrm{mg} / \mathrm{ml}$; Sigma, St. Louis, MO) to stop the trypsinization. The dissociated suspension was then centrifuged and cells were suspended with serum-free NSPC medium containing DMEM/F-12 (Invitrogen, San Diego, CA) supplemented with 2\% B27 (Invitrogen), basic fibroblast growth factor (bFGF; $20 \mathrm{ng} / \mathrm{ml}$; R\&D, Minneapolis, MN), and epidermal growth factor (EGF; $20 \mathrm{ng} / \mathrm{ml}$; R\&D), cultured in an incubator with $5 \% \mathrm{CO}_{2}$ at $37^{\circ} \mathrm{C}$. After two passages, NSPCs were used for experiments.

For identification of NSPCs, some neurospheres were collected and fixed with $4 \%$ paraformaldehyde at $4^{\circ} \mathrm{C}$ overnight followed with $20 \%$ sucrose at $4^{\circ} \mathrm{C}$ overnight for cryoprotection. Fourteen- $\mu \mathrm{m}$ thick sections were cut with a cryostat microtome and mounted onto gelatinized slides. The sections were stored at $-20^{\circ} \mathrm{C}$ for staining.

For NSPC proliferation experiment, single, dissociated cells were seeded into a 96-well plate at a density of $1 \times 10^{5} / \mathrm{ml}$ with different MIF concentration $(0,1,2,4,8,16$, 32ng/ml; R\&D, Minneapolis, MN) with or without IWR-1 (10 $\mu$ M; Sigma, St. Louis, MO). Four days later, observed the neurospheres and took photomicrographs with an invert microscope. Six pups were used in single culture and experiments were repeated 3 times. Analyzed the images by counting the number and measuring the diameter of neurospheres with Image-Pro Plus 5.0 software. Part of the cells were seeded on poly-D-lysine hydrochloride (PLL, Sigma-Aldrich, molecular weight of 70,000 $\sim 150,000$ ) - coated 10-mm glass coverslips in 24-well plates in NSPC medium and immunostained with Ki67 and Hoechst antibodies four days later. For Ki67-immunostaining cells, MIF concentration is $16 \mathrm{ng} / \mathrm{ml}$ in MIF-stimulated group.

For NSPC differentiation studies, neurospheres were seeded on poly-D-lysine hydrochloride (PLL, Sigma-Aldrich, molecular weight of 70,000 150,000) - coated 10-mm glass coverslips in 24-well plates or flasks with neural differentiation medium containing DMEM/F-12 supplemented with 2\% B27 and 2\% fetal bovine serum (FBS; Invitrogen), with or without MIF $(16 \mathrm{ng} / \mathrm{ml})$ or IWR-1 (1 or $10 \mu \mathrm{M})$. Seven to ten days later, stopped differentiation and fixed the cells with $4 \%$ paraformaldehyde for staining or collected the cells in RIPA buffer for Western blot.

\section{Immunocytochemistry}

Immunostaining was performed with the following primary antibodies and dilutions: rab- bit-anti-nestin (1:500, Sigma, St. Louis, MO, USA) for identification of NSPCs; rabbit-anti-Ki67 (1:1000, Abcam, UK) for proliferating NSPCs; rabbit anti- $\beta$-catenin (1:1000, Sigma) for identification of nuclear $\beta$-catenin; rabbit-anti-doublecortin (DCX, 1:1000, Sigma) and mouse-anti-Tuj 1 (also known as beta-tubulin III, 1:1000, Sigma) for neuron; rabbit-anti-glial fibrillary acidic protein (GFAP, 1:4000, Chemicon, Temecula, CA, USA) for astrocyte; rabbit-anti-CNPase (1:500, Sigma) for oligodendrocyte; rabbit-anti- $\beta$-galactosidase (1:2000, Chemicon, Temecula, CA, USA) for $\beta$-galactosidase expression. As secondary antibodies, the Alexa Fluor 488 or Alexa Fluor 568 - labeled secondary antibody (1:500, Molecular Probes Inc., Eugene, OR, USA) were used. Nuclei were counterstained with Hoechst 33258 (1 ng/mL; Sigma).

Fixed cells and neurosphere sections were incubated with primary antibody in $0.01 \mathrm{M}$ PBS containing $1 \%$ bovine serum albumin at $4^{\circ} \mathrm{C}$ overnight followed by incubation with an appropriate secondary antibody for 1 hour at room temperature. Nuclei were counterstained with Hoechst 33258 for 10 min at room temperature. Washing samples after each step with 0.01M PBS for three times. Cells were visualized and taken the photomicrographs with fluorescent microscope (BX-51, Olympus, Tokyo, Japan) or confocal laser scanning microscopy (FV1000, Olympus, Tokyo, Japan). Negative controls that were processed in the absence of primary antibodies did not show unspecific staining (data not shown).

\section{Western blot}

The standard procedure for Western blot was used as previously described [26]. Briefly, differentiated NSPCs were harvested in RIPA buffer containing $2 \%$ protease inhibitor (Boehringer Mannheim, Mannheim, Germany). Cell lysates were loaded onto $10 \%$ polyacrylamide gel, and then transferred to PVDF membranes (Millipore, Billerica, MA). The membranes were probed with primary antibody (rabbit-anti-DCX, 1:1000, Sigma; mouse anti-Tuj1, 1:1000, Sigma; rabbit anti- $\beta$-catenin, 1:1000, Sigma; goat anti-Wnt1, 1:1000, Sigma; rabbit anti- $\beta$-actin, 1:3000, sigma) and second antibody (horseradish peroxidase-conjugated goat anti-mouse or rabbit secondary antibody, Jackson ImmunoResearch, West Grove, PA) successively using standard method and developed with ECL kit (Millipore) according to the manufacturer's instruction. The intensity of immunoblotting bands were analyzed using Image-Pro Plus 5.0 software. Band intensities of Tuj1, DCX, $\beta$-catenin and Wnt1 were normalized to those of corresponding loading control ( $\beta$-actin). 


\section{Statistic}

Results are all shown as mean \pm S.E. All analyses were performed with statistical software SPSS (version 13.00). The differences between two groups were evaluated by Independent Samples $t$-test. The differences among multiple groups were assessed by one-way or two-way ANOVA followed by a post-hoc $t$ test. The criterion for statistical significance was $p<0.05$.

\section{Results}

\section{Neuropheres are nestin-positive and capable of differentiating to neuron, astrocyte and ol- igodendrocyte.}

For identification of NSPCs, the sections of neurospheres after two passages were stained with nestin (a marker of NSPCs). Nuclei were counterstained with Hoechst 33258. The result showed that the majority of the cells were nestin-positive (Fig. 1). To further essay the multipotent capacity of NSPCs to differentiate into neuron and glia, neurospheres were cultured on PLL-coated coverslips in differentiation medium for 4-14 days, and then immunostained with Tuj 1 antibody for neuron, GFAP for astrocyte, and CNPase for oligodendrocyte. The results showed that all of the three types of cells were stained out with different cell morphology (Fig. 1), which indicated the multipotency of these NSPCs.

\section{MIF enhances NSPC proliferation.}

In our previous studies, we found that MIF was capable of promoting the proliferation of embryonic stem cells [21]. Therefore, in the present study, we wonder whether MIF has the capacity of enhancing NSPCs self-renewal. We analyzed the expression of Ki67 indicating the proliferating cells, and the size and number of formed neurospheres followed MIF stimulation_(16ng/ml). The results showed that there were more Ki67-expressing cells in MIF-stimulation groups than those in control groups (Fig. 2A, $p<0.05$, $\mathrm{n}=3$ animals in each group). There was no evident difference of neurosphere numbers between control and MIF-stimulation groups. However, with MIF stimulation, the mean volume of neurospheres was evidently larger than that in control group without MIF (Fig. 2B, $p<0.0001, n=6$ wells in each group, repeated 3 times). Moreover, MIF promoted NSPC proliferation in a dose-dependent manner in vitro, an effect that was highly significant. The maximum effect appeared when MIF concentration was $16 \mathrm{ng} / \mathrm{ml}$. The mean volume of neurospheres treated with $16 \mathrm{ng} / \mathrm{ml}$ of MIF was more than two times as that in control group without MIF. These results suggest that NSPCs proliferate more rapidly with MIF stimulation than that without MIF stimulation.
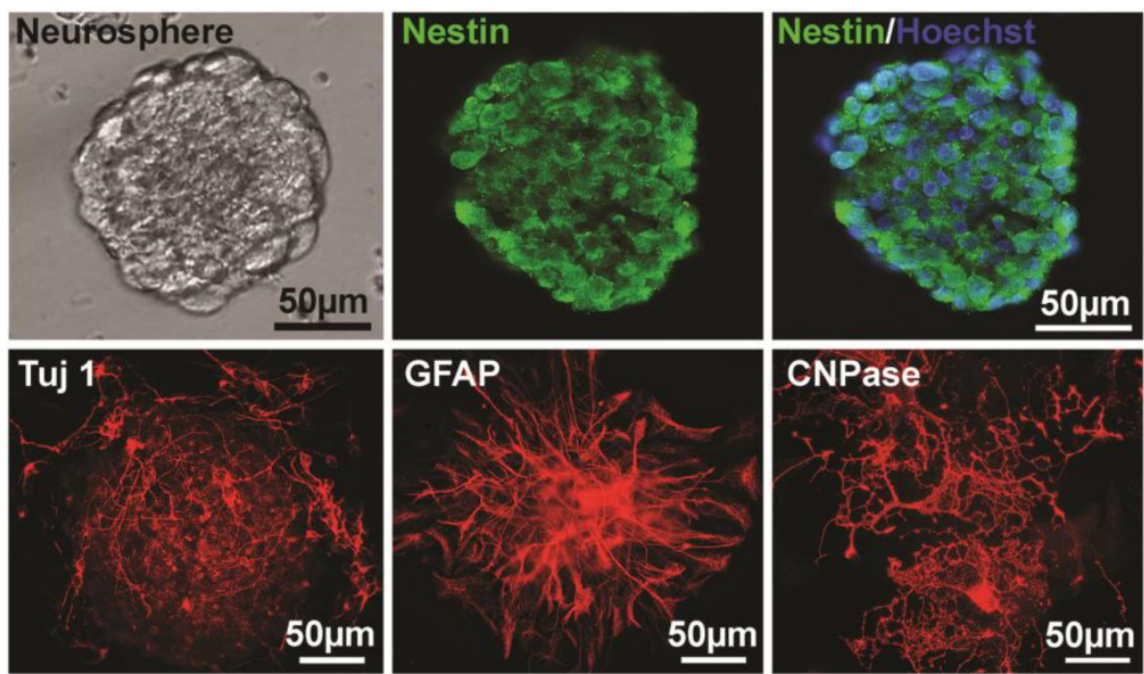

Figure I. Identification of NSPCs (neurospheres) derived from mouse brain. The left image of the upper panel shows the cultured NSPCs grow in a manner of neurospheres. The middle and the right of the upper panel show that cells of neurospheres are Nestin-positive and Nestin/Hoechst double staining, respectively. The lower panel shows the Tuj I- (for neuron, 4 days after differentiation), GFAP- (for astrocyte, 4 days after differentiation), and CNPase- (for oligodendrocyte, I0 days after differentiation) positive cells differentiated from neurospheres, respectively. 
A
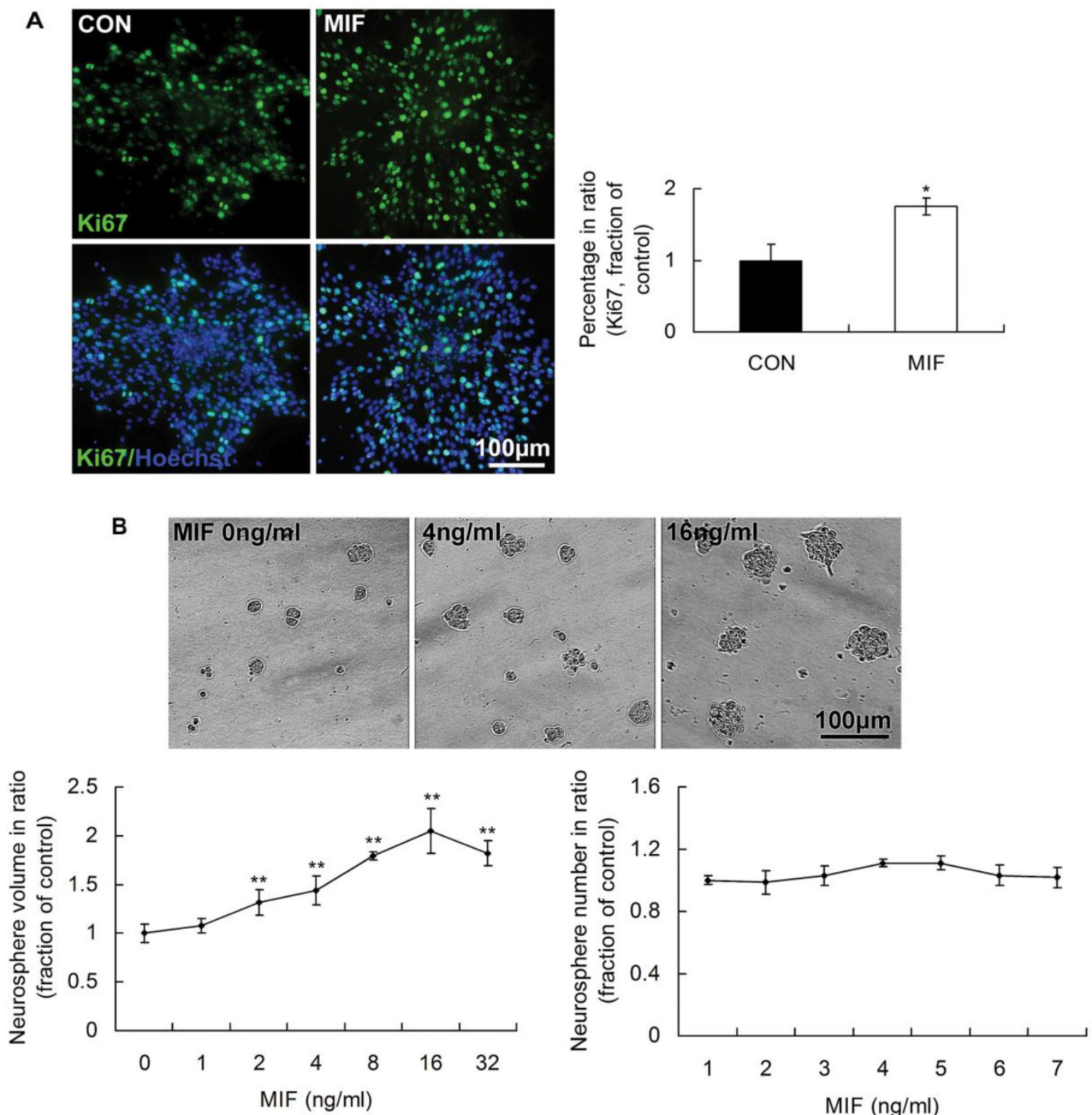

Figure 2. The promoting effects of MIF on NSPC proliferation. A. Representative images of immunostaining show Ki67-postive cells (upper panel) and Ki67/Hoechst double staining cells (lower panel) in control and MIF-stimulated groups (MIF I6ng/ml); and the comparison of Ki67-positive cell percentages between control and MIF-stimulated groups (" $p<0.05$ vs. control group, $n=3$ animals in each group). B. Representative images show neurospheres incubated with different concentration of MIF (upper panel); and comparisons of the neurosphere volumes and numbers at different concentration of MIF by ANOVA (** $p<0.000 \mathrm{I}$ vs. MIF $0 \mathrm{ng} / \mathrm{ml}$ group, $\mathrm{n}=6 \mathrm{wells}$ in each group, repeated 3 times) (lower panel). CON, control group; MIF, MIF-stimulated group.

\section{MIF enhances NSPC proliferation through Wnt/ $\beta$-catenin pathway.}

It was reported that $\beta$-catenin signaling plays a role in the proliferation of progenitor cells in the SVZ of adult mice. The activation of canonical Wnt signal pathway promotes neural precursor proliferation in vivo [22]. However, the interactions between MIF and Wnt/ $\beta$-catenin signal pathways, as well as the functions of their interactions, in neurogenesis remain largely unstudied. So, we wonder whether $W n t / \beta$-catenin is involved in transducing MIF signal of promoting NSPC proliferation or not. The immunostaining results showed that the expression of $\beta$-catenin in nuclei was stronger in MIF-stimulated group than that in control group (Fig. 3A, $p<0.05, \mathrm{n}=3$ animals in each group), indicating the activation of Wnt/ $\beta$-catenin signaling. Western blot result further proved the increase of $\beta$-catenin with MIF stimulation indicating the activation of $\mathrm{Wnt} / \beta$-catenin signaling (Fig 3B, $p<0.0001, \mathrm{n}=3$ animals in each group). Furthermore, IWR-1 $(10 \mu \mathrm{M})$ was used here to inhibit Wnt/ $\beta$-catenin pathway. IWR-1 induces stabilization of $\beta$-catenin disruption complex, the APC/Axin2/GSK3b complex, decreases the cytoplamic free $\beta$-catenin level via a direct interaction, and works as a Wnt/ $\beta$-catenin signal pathway inhibitor [27]. Four days after culture, the size of formed neu- 
rospheres was increased in a dose-dependent manner $(0,4$ and $16 \mathrm{ng} / \mathrm{ml})$. However, once IWR-1 $(10 \mu \mathrm{M})$ was added to the medium together with MIF, the size of cell colonies was extremely decreased compared to those only with MIF (Fig 3C, $p<0.0001, \mathrm{n}=6$ wells in each group, repeated 3 times), which indicated the promoting effect of MIF on NSPC proliferation was inhibited by IWR-1 in any MIF concentration group. Although these neurospheres in all groups continued growth after 7 days or even longer, the effect of IWR-1 was the same (data not shown). The effect of IWR-1 alone on NSPC proliferation was also evaluated. NSPCs were incubated with different concentration of IWR-1 individually. The results showed that 2, 5 and $10 \mu \mathrm{M}$ of IWR-1 significantly inhibited the proliferation of NSPC dose-dependently (Fig. 3D, $p<0.05, \mathrm{n}=8$ wells in each group, repeated 3 times). These results suggest that Wnt/ $\beta$-catenin pathway is involved in MIF promoting effect on NSPC proliferation.

\section{MIF promotes the differentiation of NSPCs to neuron lineage}

The above results suggest that MIF can enhance the proliferation of NSPCs. However, the effect of MIF on NSPCs differentiation is largely unknown. In this experiment, we studied the effect of MIF on NSPC differentiation into neuron by immunostaining and Western blot. DCX and Tuj1 were used as neuron markers. MIF $(16 \mathrm{ng} / \mathrm{ml})$ was added to NSPC differentiation medium in MIF-stimulated group. After 7-10 days differentiation, cells were subjected to immunostaining or Western blot assay. Immunostaining results showed that lots of cells extended long DCX-positive processes in both control and MIF-stimulated groups. But in MIF-stimulated group, there were much more DCX-positive processes than those in control group (Fig. 4A, $p<0.0001$, n=3 animals in each group). Western blot results further proved the upregulation of DCX with MIF stimulation ((Fig. $4 \mathrm{~B}, p<0.0001, \mathrm{n}=3$ animals in each group). Another neuron marker, Tuj1, showed the similar results (Fig 4C). Some Tuj1-positive cells were stained out in both control and MIF-stimulated groups. However, in MIF-stimulated group, there are much more Tuj1-positive cells than those in control group. Moreover, the number of migrated Tuj1-positive cells out from the neurospheres was much higher in MIF-stimulated group than those in control group (Fig. $4 C, p<0.05, n=3$ animals in each group). Western blot result also showed the upregulation of Tuj1 expression with MIF stimulation ((Fig. $4 \mathrm{D}, p<0.05$, $\mathrm{n}=3$ animals in each group). Taken together, these results strongly suggested that MIF not only promoted NSPC differentiation into neuron lineage but also promoted the neuronal migration from the neurospheres.
Wnt/ $\beta$-catenin signal pathway is involved in the event that MIF promote NSPC differentiation to neuron linage.

MIF activated Wnt/ $\beta$-catenin signal pathway during NSPC differentiation indicated with TOPGAL transgenic mice

LacZTCF $\mathrm{Tg}$ mice, in which $\beta$-galactosidase expresses in the presence of the lymphoid enhancer binding factor $1 /$ transcription factor 3 (LEF/TCF) mediated signaling pathway and activated $\beta$-catenin, were used to indicate the activation of $\mathrm{Wnt} / \beta$-catenin signal pathway [28]. In this experiment, single cells dissociated from neurospheres from two-day-old pups of LacZ ${ }^{\mathrm{TCF}} \mathrm{Tg}$ mice were seeded on PLL-coated coverslips and cultured with NSPC differentiation medium. MIF (16ng/ml) was added to NSPC differentiation medium in MIF-stimulated group. After 2 days culture, cells were stained with anti- $\beta$-galactosidase antibody to track the activation of $W n t / \beta$-catenin signal pathway in individual cell. Immunostaining results showed that $\beta$-galactosidase-positve cells appeared in both of the control and MIF-stimulated groups. In control group, $\beta$-galactosidase-positve products mainly located in and around the nuclei. However, in MIF-stimulated group, there were much more $\beta$-galactosidase-positve products appeared in the cytoplasm around the nuclei and the processes than those in control group (Fig. $5 \mathrm{~A})$. $\beta$-galactosidase expression evidently increased in MIF-stimulated group in comparison with that in control group (Fig. 5A, $p<0.05, \mathrm{n}=3$ animals in each group), which indicated the activation of Wnt/ $\beta$-catenin signal pathway with MIF stimulation during NSPC differentiation.

$\beta$-catenin and Wntl are upregulated with MIF stimulation during NSPC differentiation

Our results have showed that MIF can activate Wnt/ $\beta$-catenin pathway indicated with TOPGAL transgenic mice, but the cellular contributors and signaling pathways through which MIF may exert its direct action on NSPCs have not yet been identified. $\beta$-catenin is the key player in Wnt/ $\beta$-catenin signal pathway, and the amount of $\beta$-catenin can be upregulated by Wnt signaling. Wnt1 is the prototypical ligand of $W n t / \beta$-catenin pathway, and signals into the cells via the canonical $\mathrm{Wnt} / \beta$-catenin pathway [28]. Then, in this part, $\beta$-catenin and Wnt1 levels were detected by using Western blot method. The results showed that Wnt 1 and $\beta$-catenin levels in the differentiated NSPCs were evidently upregulated 2 days after MIF stimulation compared with those of control (Fig. 5B, $p<0.05, n=3$ animals in each group). These results further suggest that MIF activates 
Wnt/ $\beta$-catenin pathway during NSPC differentiation, possibly by inducing Wnt1 secretion from NSPCs and then increasing $\beta$-catenin level in the NSPCs.
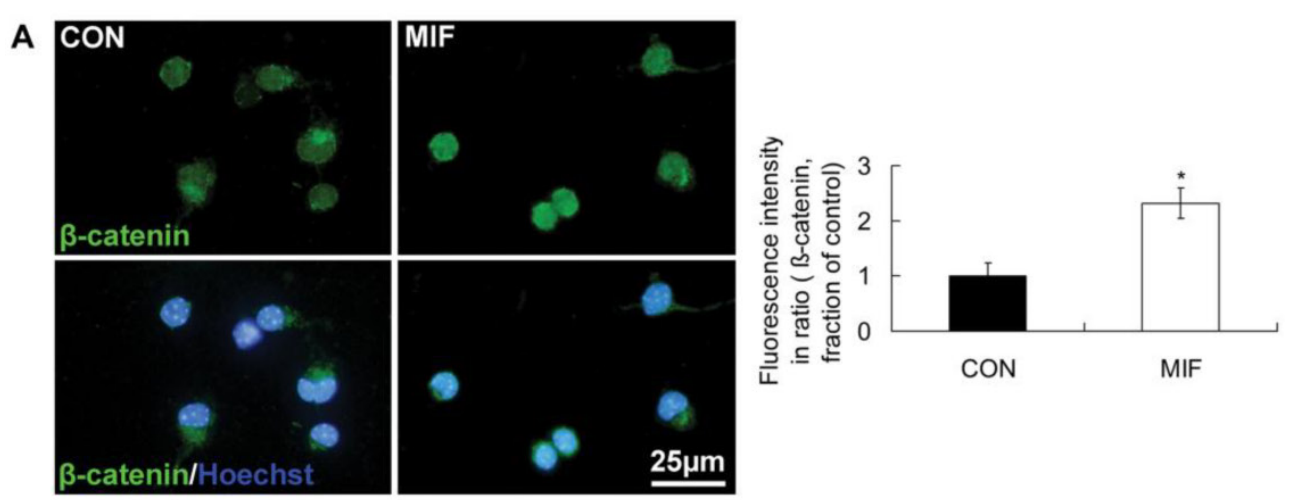

Figure 3. The involvement of Wnt/ $\beta$-catenin pathway in NSPC proliferation induced by MIF. A. Representative images of immunostaining show $\beta$-catenin-postive cells (upper panel) and $\beta$-catenin/Hoechst double staining cells (lower panel) in control and MIF-stimulated groups; and the comparison of nuclear $\beta$-catenin fluorescence intensity between control and MIF-stimulated groups $\left({ }^{*} p<0.05\right.$ vs. control group, $n=3$ animals in each group). B. Western blot results show the protein bands of $\beta$-catenin in NSPCs and the comparison of Western blot result showing the relative $\beta$-catenin levels in control and MIF-stimulated groups $(* * p<0.0001$ vs. control group, $n=3$ animals in each group). Results were normalized to $\beta$-actin. CON, control group; MIF, MIF-stimulated group.

C. Representative images show neurospheres incubated with different concentration of MIF, with or without IWR-I (upper panel); and the comparisons of neurosphere volumes among different groups by ANOVA $\left({ }^{*} p<0.05,{ }^{* *} p<0.0001\right.$ $\mathrm{n}=6$ wells in each group, repeated 3 times) (lower panel). D. The volume comparisons of neurospheres incubated with different concentration of IWR-I by ANOVA $(" p<0.05, n=8$ wells in each group, repeated 3 times)

B

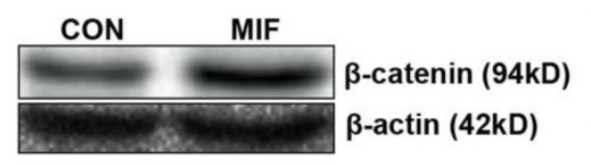

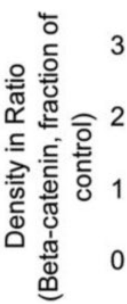

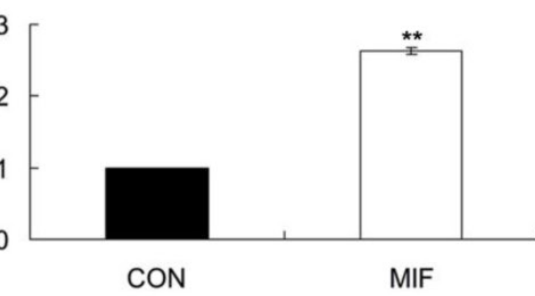

C
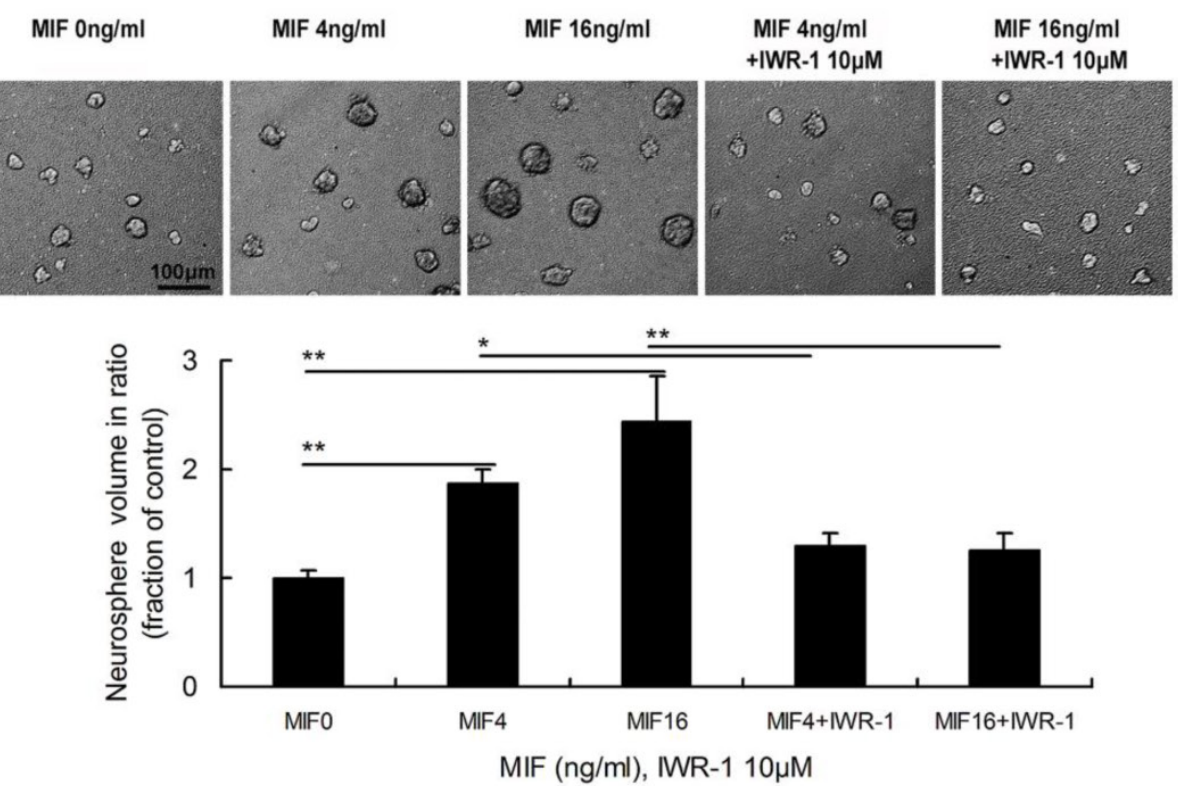

D

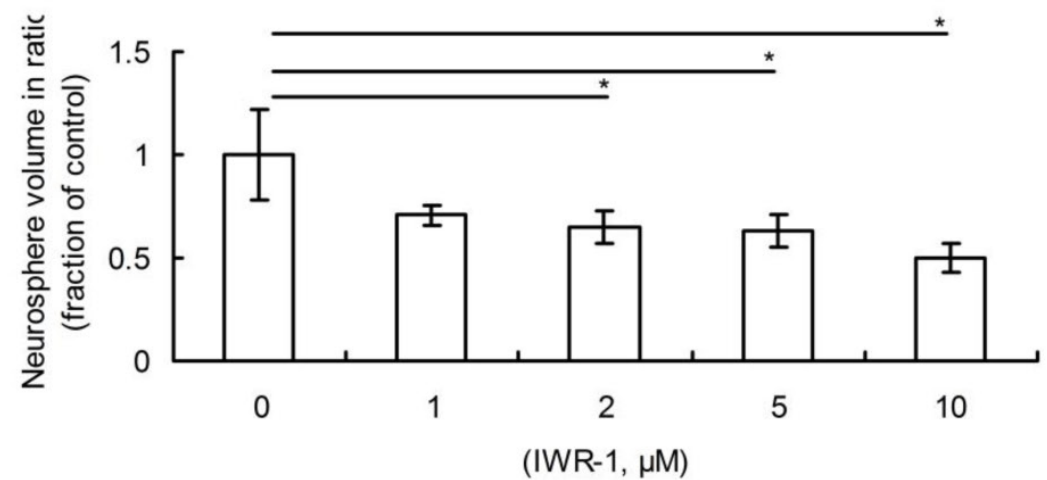


Figure 4. MIF promotes NSPC differentiation to neuron lineage. A. Representative images of immunostaining show DCX-positive cells in control and MIF-stimulated groups (MIF $16 \mathrm{ng} / \mathrm{ml}$ ) and the comparison of the areas of DCX-positive cells between control and MIF-stimulated groups $\left({ }^{*} p<0.05\right.$ vs. control group, 7 days after differentiation, $n=3$ animals in each group). The inserts at the top right corners are high magnification of the corresponding areas in the boxes in the images, respectively. B. Western blot results show the protein bands of DCX in differentiated NSPCs and the comparison of Western blot result showing the relative DCX levels in control and MIF-stimulated groups $\left({ }^{*} p<0.05\right.$ vs. control group, 7 days after differentiation, $n=3$ animals in each group). Results were normalized to $\beta$-actin. C. Representative images of immunostaining show Tuj I-positive cells in control and MIF-stimulated groups (MIF $16 \mathrm{ng} / \mathrm{ml}$ ) and the comparisons of the total Tuj I-positive cells and the Tuj I-positive cells migrated out from neurospheres between control and MIF-stimulated groups. ( $p<0.05$ vs. control group, 7 days after differentiation, $n=3$ animals in each group). The inserts at the top right corners are high magnification of the corresponding areas in the boxes in the images, respectively. D. Western blot results show Tuj I protein bands in differentiated NSPCs and the comparison of Western blot result showing the relative Tuj I levels in control and MIF-stimulated groups $(* p<0.05$ vs. control group, 7 days after differentiation, $\mathrm{n}=3$ animals in each group). Results were normalized to $\beta$-actin. CON, control group; MIF, MIF-stimulated group.
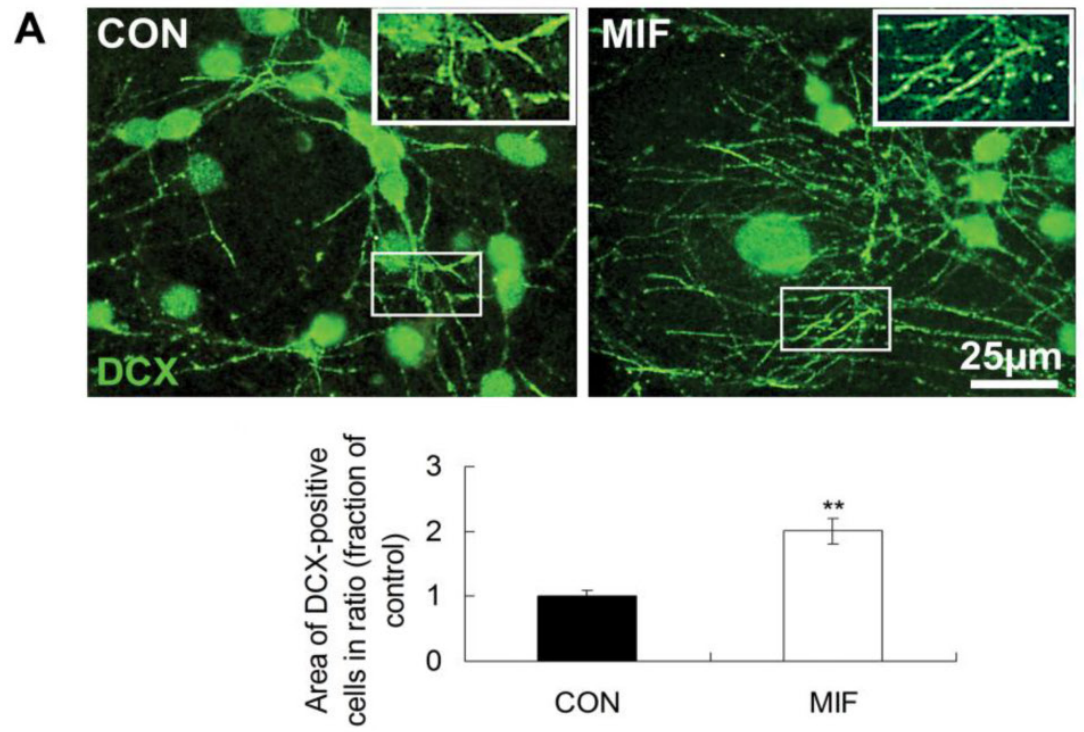

B
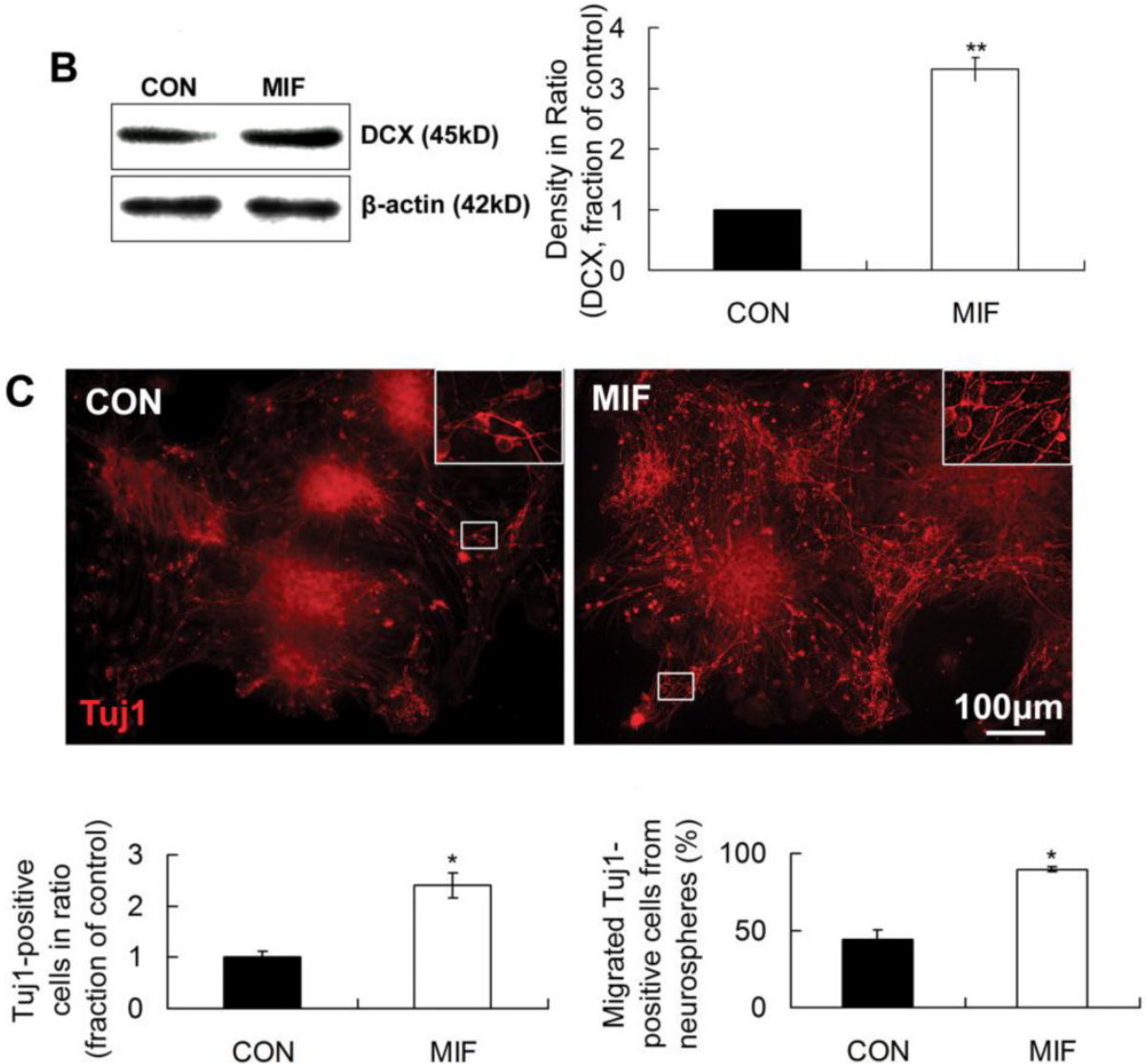

D

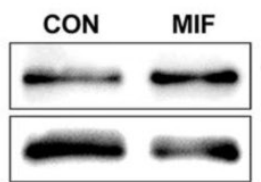

Tuj 1 (55kD)

$\beta$-actin (42kD)

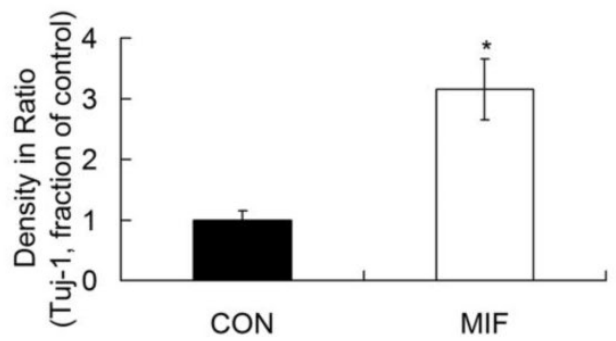



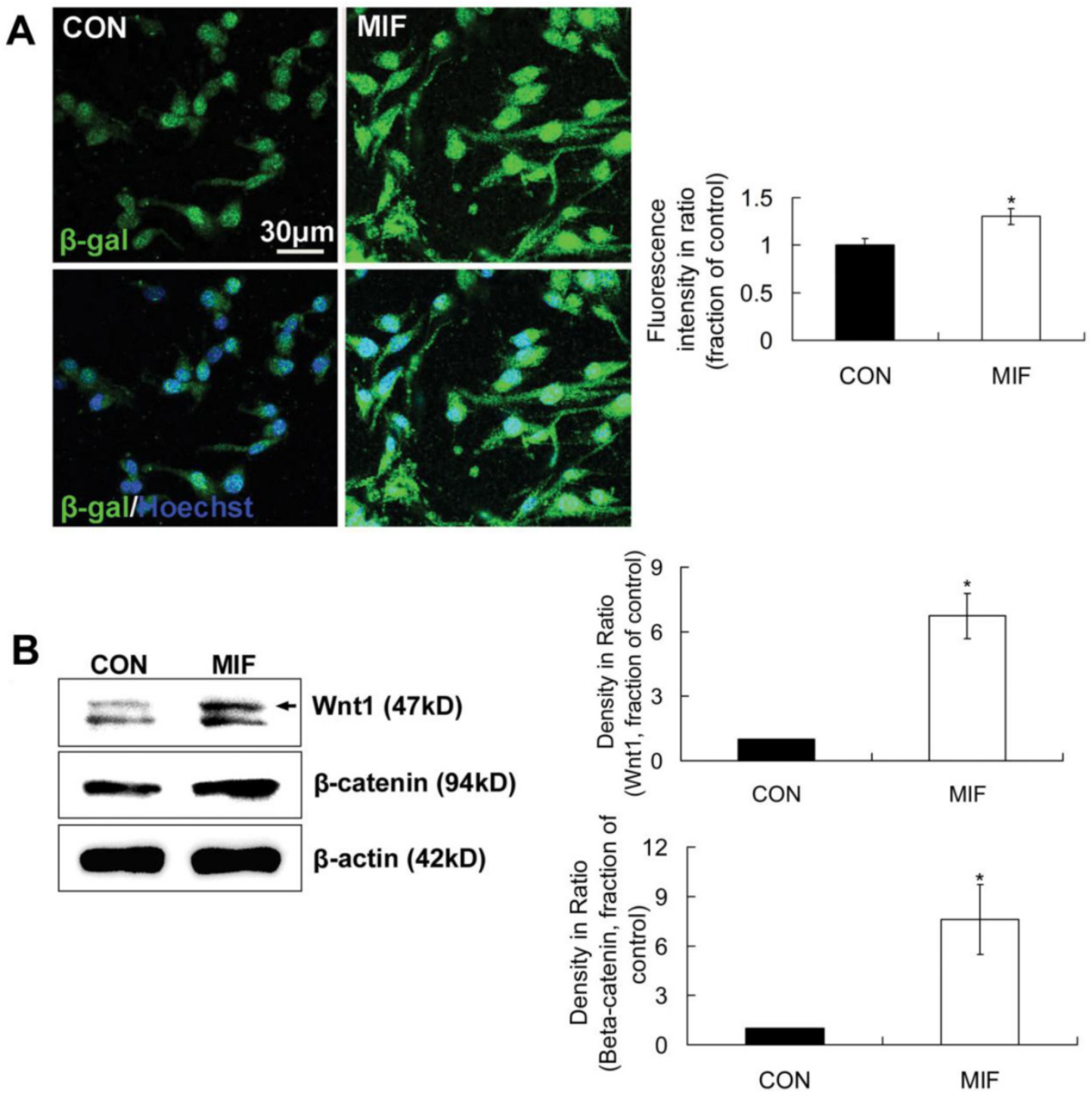

Figure 5. The activation of Wnt/ $\beta$-catenin signal pathway by MIF during NSPC differentiation. A. Representative images show $\beta$-galactosidase-positive cells (upper panel) and $\beta$-galactosidase /Hoechst double staining cells (lower panel) and the comparison of $\beta$-galactosidase-positive fluorescence intensities between control and MIF-stimulated groups ( $" p<0.05$ vs. control group, 2 days after differentiation, $n=3$ animals in each group). B. Western blot results show Wntl and $\beta$-catenin protein expressions in differentiated NSPCs in control and MIF-stimulated groups and the comparison of Western blot results showing the relative Wntl and $\beta$-catenin levels in control and MIF-stimulated groups (" $p<0.05$ vs. control group, 7 days after differentiation, $n=3$ animals in each group). CON, control group; MIF, MIF-stimulated group; $\beta$-gal, $\beta$-galactosidase

IWR-I inhibites the promoting effect of MIF on NSPC differentiation to neuron lineage.

MIF promotes NSPC differentiation to neuron lineage, and $\mathrm{Wnt} / \beta$-catenin signal pathway is activated by MIF stimulation in this event. However, whether activated $\mathrm{Wnt} / \beta$-catenin signal pathway mediating the promoting effect of MIF on NSPC differentiation to neurons or not still needs to be further investigated. Here, IWR-1 was used to inhibit Wnt/ $\beta$-catenin signal pathway [27] during NSPC differentiation. Different concentration $(1 \mu \mathrm{M}$ or $10 \mu \mathrm{M})$ of IWR-1 was added to the differentiation medium. NSPC cells were divided into 4 groups $(n=3$ animals in each group): control group with normal differentiation medium; MIF-stimulated group with MIF (16ng/ml) added; IWR-1 group only with IWR-1
$(1 \mu \mathrm{M}$ or $10 \mu \mathrm{M})$ in differentiation medium; MIF+IWR-1 group with both MIF $(16 \mathrm{ng} / \mathrm{ml})$ and IWR-1 $(1 \mu \mathrm{M}$ or $10 \mu \mathrm{M})$. Neurospheres were seeded on PLL-coated coverslips or flasks and cultured in differentiation medium for differentiation.

In the first experiment, $1 \mu \mathrm{M}$ of IWR-1 was used. Seven days after differentiation, cells were stained with anti-DCX and anti-Tuj 1 to observe the neuron lineage differentiation. The results showed that some DCX- and Tuj 1-positive cells were stained out in all of the four groups. There are much more DCX- and Tuj1-positive cells in MIF-stimulated group than those in control group (Fig. 6A, C, $p<0.0001$ ). However, with the treatment of IWR-1 $(1 \mu \mathrm{M})$, although still with MIF stimulation, DCX and Tuj 1 expression was decreased significantly (Fig. 6A, C, $p<0.0001$ ). 
Figure 6. Effects of MIF and IWR-I on neuronal differentiation of NSPC. A. Representative images of DCX immunostaining show the effect of I $\mu$ M of IWR-I on DCX expression and the comparison of DCX expression among the 4 groups by ANOVA $(* * 000001, n=3$ animals in each group $)$. The arrows show some of the DCX-positive processes. B. Representative images of DCX immunostaining show the effect of $10 \mu \mathrm{M}$ of IWR-I on DCX expression and the comparison of DCX expression among the 4 groups by ANOVA $(* *<0.0001$, $\mathrm{n}=3$ animals in each group). The arrows show some of the DCX-positive processes. C. Representative images of Tuj I immunostaining show the effect of I $\mu$ M of IWR-I on TujI expression and the comparison of Tuj I-positive cells among the 4 groups by ANOVA $\left({ }^{* * *} p<0.000 \mathrm{I}, \mathrm{n}=3\right.$ animals in each group). D. Representative images of Tuj I immunostaining show the effect of I $0 \mu$ M of IWR-I on Tuj I expression and the comparison of Tuj I-positive cells among the 4 groups by ANOVA (*** $p<0.000 \mathrm{I}, \mathrm{n}=3$ animals in each group). CON, control group; MIF, MIF-stimulated group. E. Western blot results show DCX and Tuj I protein expressions in differentiated NSPCs in control and MIF-stimulated groups with or without IWR-I (IOHM) and the comparison of Western blot results showing the relative DCX and Tuj I levels in four groups $(* *<<0.001,7$ days after differentiation, $\mathrm{n}=3$ animals in each group). CON, control group; MIF, MIF-stimulated group.
A

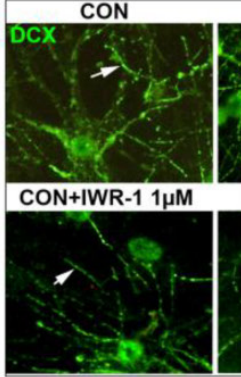

MIF
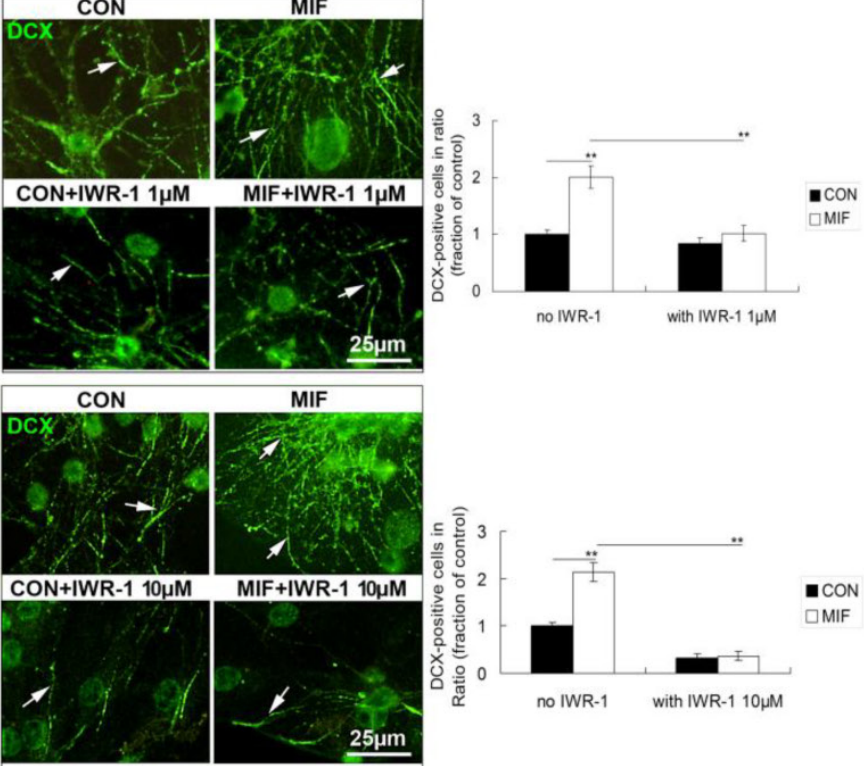

C
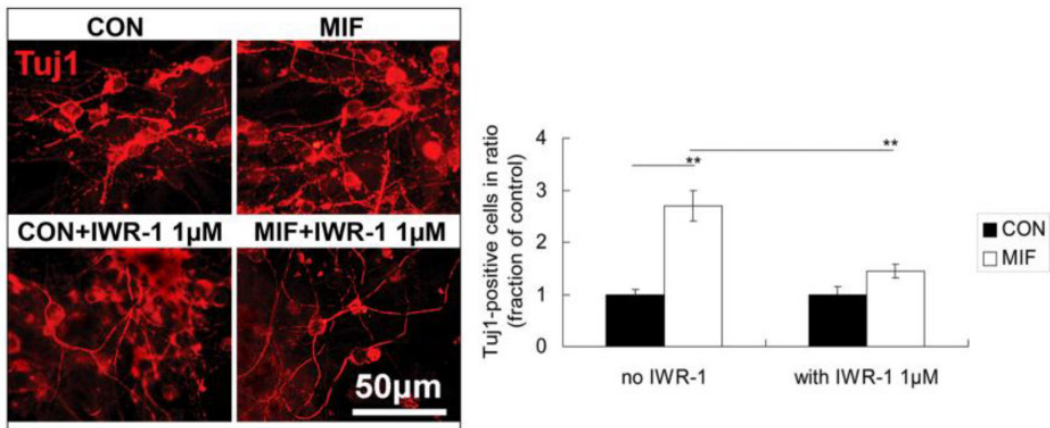

D

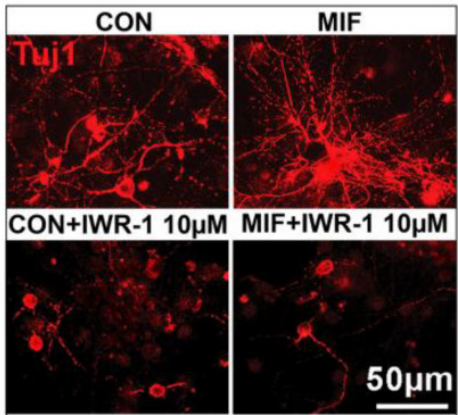

no IWR-1

with IWR-1 $1 \mu \mathrm{M}$$$
\text { . }
$$

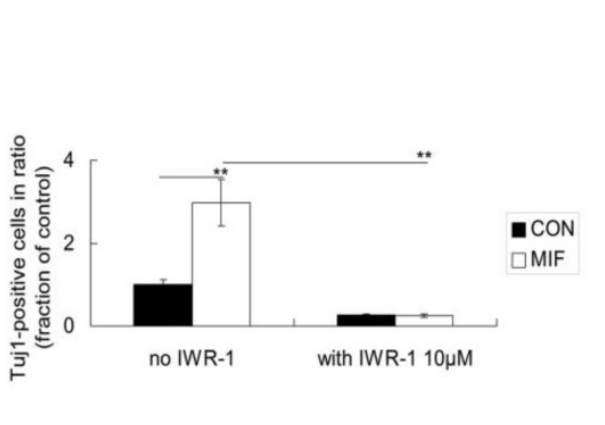

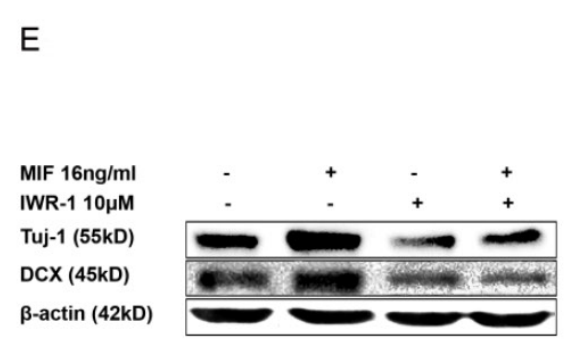

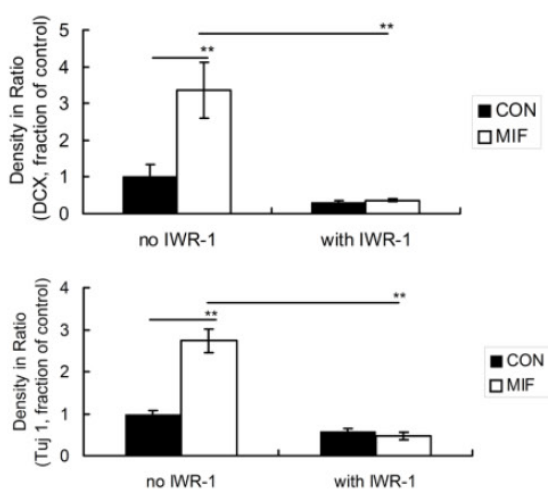


In the second experiment, $10 \mu \mathrm{M}$ of IWR-1 was tested. The results showed a stronger inhibition of $10 \mu \mathrm{M}$ of IWR-1 in MIF-induced neuronal differentiation than that of $1 \mu \mathrm{M}$ of IWR-1. DCX and Tuj 1 expression was strongly inhibited in either IWR-1 alone group or MIF+IWR-1 group (Fig. 6B, D, $p<0.0001$ ).

Western blot results further proved that $10 \mu \mathrm{M}$ of IWR-1 inhibited the expression of DCX and Tuj 1 both in control and MIF-stimulated groups (Fig. 6E, $p<0.0001)$.

These data strongly suggest that Wnt/ $\beta$-catenin signal pathway is involved in the event of MIF enhancing the neuronal differentiation from NSPCs.

\section{Discussion}

A number of trophic factors or molecules regulate NSPCs self-renewal and differentiation in vivo and in vitro. A classical point of view indicated that most of these mediators did not correspond to immune-system-derived molecules. However, growing evidence indicates that immunological molecules can target neurogenic niches and control NSPC proliferation, survival, differentiation and migration [4]. MIF is one of the important pro-inflammatory cytokines and can act as an immune regulator, an anterior pituitary hormone, high activity enzyme, a regulator of neuronal degeneration-regeneration and is well known for its role in many inflammation-based diseases [11, 16-19, 29]. However, its role in NSPC self-renewal and differentiation is largely unclear. In this study, we have shown that (i) MIF enhances NSPC proliferation; and $\mathrm{Wnt} / \beta$-catenin signal pathway is involved in this event as demonstrated by increased nuclear $\beta$-catenin with MIF stimulation and decreased neurosphere size with Wnt/ $\beta$-catenin signal pathway inhibitor, IWR-1, added; (ii) MIF promotes the differentiation and migration of neuron lineage from NSPCs; (iii) MIF upregulates the activity of LacZ ${ }^{\mathrm{TCF}}$ reporter transgene that responds to the activation of $\mathrm{Wnt} / \beta$-catenin signal pathway and the levels of Wnt1 and $\beta$-catenin proteins during NSPC differentiation. (iiii) Furthermore, inhibition of Wnt/ $\beta$-catenin signal pathway with IWR-1 significantly reduces the neuronal differentiation from NSPCs as evidenced by decreased DCX- and Tuj1- expression. This is the first study to show that MIF promotes not only the NSPC self-renewal but also the neuronal differentiation from NSPCs via $W n t / \beta$-catenin signal pathway in vitro. These new findings reveal an essential role of MIF under normal or pathological condition and a function link between MIF and Wnt/ $\beta$-catenin signal pathway in NSPCs proliferation and differentiation processes.

MIF is ubiquitously expressed in various types of the neural cells in the CNS $[10,11]$. Astrocyte, mi- croglia, neuron and NSPC, which are the main cells in the CNS, are all proved expressing MIF [10-12]. In the physiological condition, there is significant level of baseline MIF expression in the brain [10]. In many neurodegenerative disease and the CNS injury, such as Alzheimer's disease and spinal cord injury, MIF expression is increased [11, 30]. Most significantly, MIF mRNA is present in the developing neuraxes and nervous systems of mouse [31] and is a critical player in establishing the neuraxis of the embryo [32]. Moreover, NSPCs express MIF receptors, CD44, CXCR2, CXCR4 and CD74, which suggests that MIF can maintain NSPCs through autocrine and/or paracrine mechanisms [12]. These reports together with our findings indicate that MIF may be effective for the neurogenesis in physiological condition, developing brain, CNS injury and neurodegenerative diseases.

In this study, we prove that MIF is capable of stimulating the proliferation of NSPCs from two-day-old mice in a dose dependent manner. This finding is in consistent with the recent report by Ohta et al. [12]. Ohta et al. identifies MIF as a proliferation factor for NSPCs from the embryonic day 14.5 (E14.5) mice by using higher concentration of MIF than that we use. MIF is a key player in various cell proliferation, such as tumor cell growth, angiogenesis [33], keratinocytes proliferation [34], embryonic stem cell proliferation [21], and adult hippocampal cell proliferation [16]. From this study, we conclude that MIF is also a key player in control of NSPC proliferation. Moreover, we further prove that MIF enhances NSPC proliferation through the activation of $\mathrm{Wnt} / \beta$-catenin signal pathway. With MIF stimulation, the nuclear $\beta$-catenin is increased evidently. By using IWR- 1 , a Wnt/ $\beta$-catenin signal pathway inhibitor, NSPC proliferation stimulated by MIF is inhibited significantly. It has been proved that MIF plays its roles by triggering several different cell signal pathways in various cell types. Erk and Akt pathways are two important pathways activated by MIF for cell proliferation and survival, and involved in adult NSPC proliferation [12, 35]. Although $\mathrm{Wnt} / \beta$-catenin signal pathway plays a critical role in neural development and growth and related with the proliferation of neural precursors in the SVZ [22], the interactions of MIF and Wnt/ $\beta$-catenin signal pathway, as well as the functions of their interactions, in neurogenesis remain largely unknown. Our study firstly shows that Wnt/ $\beta$-catenin signal pathway plays an important role in MIF enhancing NSPC proliferation, indicating the functional interaction between MIF and Wnt/ $\beta$-catenin signal pathway in NSPC self-renewal.

Most importantly, our current study also demonstrates that MIF is capable of guiding more NSPCs differentiating to neuron lineage during the 
differentiation stage. Much more DCX- and Tuj 1-positive cells appeared and much more neurons migrated out from neurospheres following MIF stimulation compared to control groups. A growing body of evidence indicates that pro-inflammatory and other immunological mediators are also involved in determining cell precursor fate and regulate the function of NSPCs [3]. As to MIF, although increasing evidences suggest that MIF has broad roles in the regulation of neuronal functions [11, 12, 16-20], the studies about MIF influencing NSPC fate are really few. To date, only one report by Ohta et al. [12] indicated that MIF did not change the differentiation potential of NSPCs, which is different from our results. The NSPCs they used were from E14.5 mice, not from the postnatal NSCs as what we used, and extremely higher MIF concentration $(400 \mathrm{ng} / \mathrm{ml})$ was used in their experiment than that we used in this study $(16 \mathrm{ng} / \mathrm{ml})$, which may be part of the reasons making the difference? Our work firstly reveals a new important role of MIF in neurogenesis indicated by upregulated DCX and Tuj1, two markers of immature or mature neurons.

Regarding the signal pathway, we further demonstrate that $W n t / \beta$-catenin signal pathway plays a critical role in control the cell fate of MIF-induced NSPC differentiation. In the CNS, Wnt signaling appears to be involved in the majority of the processes required to generate a fully functional neuron from an NSPC, participating in early steps, such as neural induction [36] and neural precursor proliferation [37], as well as in late processes, such as neuronal differentiation [38, 39], migration [24], axon guidance [40], synaptogenesis [41] and dendritogenesis [42]. Members of the Wnt1 class signal into the cell via the canonical $\mathrm{Wnt} / \beta$-catenin pathway. Wnt signaling inhibits GSK-3 $\beta$ activity, thus increasing the amount of $\beta$-catenin, which enters the nucleus, and associates with T-cell factor/lymphoid enhancer-binding factor transcription factors, leading to the transcription of Wnt target genes involved in cell survival, proliferation, and differentiation [28, 43]. Wnt1 is involved in dopaminergic neuron development [44]. Wnt1-lmx1a forms a novel autoregulatory loop and controlled midbrain dopamine differentiation synergistically with the SHH-FoxA2 pathway [45].The canonical $\mathrm{Wnt} / \beta$-catenin pathway plays a key role in controlling the neurogenic niche for dopamine neuron development and hippocampal neurogenesis [43, 46, 47]. However, the functional interactions of MIF and Wnt/ $\beta$-catenin signal pathway in neuronal differentiation remain unclear. In our current study, by using the LacZ ${ }^{\mathrm{TCF}} \mathrm{Tg}$ mice to indicate the activation of $W n t / \beta$-catenin signal pathway, we find that MIF evidently upregulates $\beta$-galactosidase expression during NSPC differentiation suggesting the strong activation of $\beta$-catenin signal pathway by MIF. Furthermore, levels of both $\beta$-catenin, the key player in Wnt/ $\beta$-catenin signal, and Wnt1, the prototypical ligand of $W n t / \beta$-catenin signal, are significantly increased with MIF stimulation during NSPC differentiation. This is a new finding indicating the interaction of MIF and Wnt/ $\beta$-catenin signal. Furthermore, our current data show the strong inhibition of DCX and Tuj 1 expression even in MIF-stimulated groups when $W n t / \beta$-catenin signal pathway is inhibited by IWR-1, which suggests that Wnt/ $\beta$-catenin is a key player in MIF-induced neuronal differentiation of NSPC. Our findings undoubtedly indicate the new function of MIF and the functional interaction between MIF and $W n t / \beta$-catenin signal pathway in neurogenesis.

In conclusion, this study demonstrates a new role of MIF in enhancing NSPC proliferation and neuronal differentiation, and reveals that $W n t / \beta$-catenin signal pathway is involved in these events. It has been proved that $\mathrm{Wnt} / \beta$-catenin signaling appears to be involved in the majority of the processes required to generate a fully functional neuron from an NSPC, participating in early steps, such as neural precursor proliferation [37] and neural induction [36], as well as in late processes, such as neuronal differentiation $[38,39]$ in the CNS. According to our results, we speculate that MIF and Wnt/ $\beta$-catenin may play different role depending on different development periods in vivo or different culture condition in vitro.

Our findings strongly point to an important role of MIF and the functional interaction between MIF and $\mathrm{Wnt} / \beta$-catenin signal pathway in neurogenesis. MIF has the potential to be used to modulate NSPC renewal and fate in an attempt to produce and control NSPCs for regenerative therapies.

\section{Abbreviations}

bFGF, basic fibroblast growth factor; DCX, doublecortin; EGF, epidermal growth factor; GFAP, glial fibrillary acidic protein; IL, interleukin; MIF, macrophage migration inhibitory factor; NSPCs, neural stem/progenitor cells; SGZ, subgranular zone; SVZ, subventricular zone; TNF, tumor-necrosis factor

\section{Acknowledgement}

We thank Haifeng Zhang, who works in Institute of Neurosciences, the Fourth Military Medical University, for help with confocal laser scanning microscopy. We also thank Prof. Yan Lu, who works in the Department of Anesthesiology and Pain Management, Xijing Hospital, the Fourth Military Medical University, for his kindly English editing. This work 
was supported by grants from the National Natural Science Foundation of China (81171156 and 81272135), NIH (R01 GM100474-01) and the New Jersey Commission on Spinal Cord Research (CSCR13IRG006).

\section{Competing Interests}

The authors have declared that no competing interest exists.

\section{References}

1. Massirer KB, Carromeu C, Griesi-Oliveira K, Muotri AR. Maintenance and differentiation of neural stem cells. Wiley Interdiscip Rev Syst Biol Med. 2011; 3: 107-14.

2. Moyse E, Segura S, Liard O, Mahaut S, Mechawar N. Microenvironmental determinants of adult neural stem cell proliferation and lineage commitment in the healthy and injured central nervous system. Curr Stem Cell Res Ther. 2008; 3: 163-84.

3. Gonzalez-Perez O, Gutierrez-Fernandez F, Lopez-Virgen V, Collas-Aguilar J, Quinones-Hinojosa A, Garcia-Verdugo JM. Immunological regulation of neurogenic niches in the adult brain. Neuroscience. 2012; 226: 270-81.

4. Bloom BR, Bennett B. Mechanism of a reaction in vitro associated with delayed-type hypersensitivity. Science. 1966; 153: 80-2.

5. Eibl MM, Sitko C. MIF production of lymphocytes from patients with rheumatoid arthritis with antigen-antibody complexes. Ann Rheum Di. 1975; 34: 117-21.

6. Bucala R. MIF, a previously unrecognized pituitary hormone and macrophage cytokine, is a pivotal mediator in endotoxic shock. Circ Shock. 1994; 44: 35-9.

7. Bucala R. Signal transduction. A most interesting factor. Nature. 2000; 408: 146-7.

8. Fingerle-Rowson GR, Bucala R. Neuroendocrine properties of macrophage migration inhibitory factor (MIF). Immunol Cell Biol. 2001; 79: 368-75.

9. Renner P, Roger T, Calandra T. Macrophage migration inhibitory factor: Gene polymorphisms and susceptibility to inflammatory diseases. Clin Infect Dis. 2005; 41:S513-9.

10. Bacher M, Meinhardt A, Lan HY, Dhabhar FS, Mu W, Metz CN, Chesney JA, Gemsa D, Donnelly T, Atkins RC, Bucala R. MIF expression in the rat brain: implications for neuronal function. Mol Med. 1998; 4: 217-30.

11. Koda M, Nishio $Y$, Hashimoto M, Kamada T, Koshizuka S, Yoshinaga K, Onodera S, Nishihira J, Moriya H, Yamazaki M. Up-regulation of macrophage migration-inhibitory factor expression after compression-induced spinal cord injury in rats. Acta Neuropathol. 2004; 108: 31-6.

12. Ohta S, Misawa A, Fukaya R, Inoue S, Kanemura Y, Okano H, Kawakami $Y$, Toda M. Macrophage migration inhibitory factor (MIF) promotes cell survival and proliferation of neural stem/progenitor cells. J Cell Sci. 2012; 125: 3210-20.

13. Leng L, Metz CN, Fang Y, Xu J, Donnelly S, Baugh J, Delohery T, Chen Y, Mitchell RA, Bucala R. MIF signal transduction initiated by binding to CD74. J Exp Med. 2003; 197: 1467-76.

14. Schwartz V, Lue H, Kraemer S, Korbiel J, Krohn R, Ohl K, Bucala R, Weber C, Bernhagen J. A functional heteromeric MIF receptor formed by CD74 and CXCR4. FEBS Lett. 2009; 583: 2749-57.

15. Shi X, Leng L, Wang T, Wang W, Du X, Li J, McDonald C, Chen Z, Murphy J. W., Lolis E, Noble P, Knudson W, Bucala R. CD44 is the signaling component of the macrophage migration inhibitory factor-CD74 receptor complex. Immunity. 2006; 25: 595-606

16. Conboy L, Varea E, Castro JE, Sakouhi-Ouertatani H, Calandra T, Lashuel HA, Sandi C. Macrophage migration inhibitory factor is critically involved in basal and fluoxetine-stimulated adult hippocampal cell proliferation and in anxiety, depression, and memory-related behaviors. Mol Psychiatry. 2011; 16: 533-47.

17. Nishio Y, Minami A, Kato H, Kaneda K, Nishihira J. Identification of macrophage migration inhibitory factor (MIF) in rat peripheral nerves: its possible involvement in nerve regeneration. Biochim Biophys Acta. 1999; 1453: 74-82.

18. Nishio Y, Nishihira J, Ishibashi T, Kato H, Minami A. Role of macrophage migration inhibitory factor (MIF) in peripheral nerve regeneration: anti-MIF antibody induces delay of nerve regeneration and the apoptosis of Schwann cells. Mol Med. 2002; 8: 509-20.

19. Nishio $\mathrm{Y}$, Koda M, Hashimoto M, Kamada T, Koshizuka S, Yoshinaga K, Onodera S, Nishihira J, Okawa A, Yamazaki M. Deletion of macrophage migration inhibitory factor attenuates neuronal death and promotes functional recovery after compression-induced spinal cord injury in mice. Acta Neuropathol. 2009; 117: 321-8.

20. Thanos S, Mey J, Wild M. Treatment of the adult retina with microglia-suppressing factors retards axotomy-induced neuronal degradation and enhances axonal regeneration in vivo and in vitro. J Neurosci. 1993; 13: 455-66.

21. Wang $X$, Chen $T$, Leng L, Fan J, Cao K, Duan Z, Zhang X, Shao C, Wu M, Tadmori I, Li T, Liang L, Sun D, Zheng S, Meinhardt A, Young W, Bucala $\mathrm{R}$, Ren Y. MIF produced by bone marrow-derived macrophages contributes to teratoma progression after embryonic stem cell transplantation. Cancer Res. 2012; 72: 2867-78.
22. Adachi $\mathrm{K}$, Mirzadeh $\mathrm{Z}$, Sakaguch $\mathrm{M}$, Yamashita $\mathrm{T}$, Nikolcheva $\mathrm{T}$, Gotoh Y, Peltz G, Gong L, Kawase T, Alvarez-Buylla A, Okano H, Sawamoto K. Beta-catenin signaling promotes proliferation of progenitor cells in the adult mouse subventricular zone. Stem Cells. 2007; 25: 2827-36.

23. Pei Y, Brun SN, Markant SL, Lento W, Gibson P, Taketo MM, Giovannini M, Gilbertson RJ, Wechsler-Reya RJ. WNT signaling increases proliferation and impairs differentiation of stem cells in the developing cerebellum. Development. 2012; 139: 1724-33.

24. Chenn A, Walsh CA. Increased neuronal production, enlarged forebrains and cytoarchitectural distortions in beta-catenin overexpressing transgenic mice. Cereb Cortex. 2003; 13: 599-606.

25. DasGupta R, Fuchs E. Multiple roles for activated LEF/TCF transcription complexes during hair follicle development and differentiation. Development. 1999; 126:4557-68.

26. Wang $X$, Wang BR, Duan $X L$, Zhang $P$, Ding $Y Q$, Jia $Y$, Jiao $X Y$, Ju G. Strong expression of interleukin-1 receptor type I in the rat carotid body. J Histochem Cytochem. 2002; 50: 1677-84.

27. Chen B, Dodge ME, Tang W, Lu J, Ma Z, Fan CW, Wei S, Hao W, Kilgore J, Williams NS, Roth MG, Amatruda JF, Chen C, Lum L. Small molecule-mediated disruption of Wnt-dependent signaling in tissue regeneration and cancer. Nat Chem Biol. 2009; 5: 100-7.

28. Marchetti B, L'episcopo F, Morale MC, Tirolo C, Testa N, Caniglia S, Serapide MF, Pluchino S. Uncovering novel actors in astrocyte-neuron crosstalk in Parkinson's disease: the Wnt/ $\beta$-catenin signaling cascade as the common final pathway for neuroprotection and self-repair. Eur J Neurosci. 2013; 37: 1550-63.

29. Nishihira J. Molecular function of macrophage migration inhibitory factor and a novel therapy for inflammatory bowel disease. Ann N Y Acad Sci. 2012; 1271: 53-7.

30. Bacher M, Deuster O, Aljabari B, Egensperger R, Neff F, Jessen F, Popp J, Noelker C, Reese JP, Al-Abed Y, Dodel R. The role of macrophage migration inhibitory factor in Alzheimer's disease. Mol Med. 2010; 16: 116-21.

31. Kobayashi S, Satomura K, Levsky JM, Sreenath T, Wistow GJ, Semba I, Shum L, Slavkin HC, Kulkarni AB. Expression pattern of macrophage migration inhibitory factor during embryogenesis. Mech Dev. 1999; 84: 153-6.

32. Suzuki M, Takamura $Y$, Maeno $M$, Tochinai $S$, Iyaguchi $D$, Tanaka I, Nishihira J, Ishibashi T. Xenopus laevis macrophage migration inhibitory factor is essential for axis formation and neural development. J Biol Chem. 2004; 279: 21406-14.

33. Shimizu T, Abe R, Nakamura H, Ohkawara A, Suzuki M, Nishihira J. High expression of macrophage migration inhibitory factor in human melanoma cells and its role in tumor cell growth and angiogenesis. Biochem Biophys Res Commun. 1999; 264: 751-8.

34. Gesser B, Rasmussen MK, Raaby L, Rosada C, Johansen C, Kjellerup RB, Kragballe K, Iversen L. Dimethylfumarate inhibits MIF-induced proliferation of keratinocytes by inhibiting MSK1 and RSK1 activation and by inducing nuclear p-c-Jun (S63) and p-p53 (S15) expression. Inflamm Res. 2011; 60: 643-53.

35. Kalluri HS, Vemuganti R, Dempsey RJ. Mechanism of insulin-like growth factor I-mediated proliferation of adult neural progenitor cells: role of Akt. Eur J Neurosci. 2007; 25: 1041-8

36. Wilson SI, Rydstrom A, Trimborn T, Willert K, Nusse R, Jessell TM, Edlund T. The status of Wnt signalling regulates neural and epidermal fates in the chick embryo. Nature. 2001; 411: 325-30.

37. Panhuysen M, Vogt Weisenhorn DM, Blanquet V, Brodski C, Heinzmann U, Beisker W, Wurst W. Effects of Wnt1 signaling on proliferation in the developing mid-/hindbrain region. Mol Cell Neurosci. 2004; 26: 101-11.

38. Muroyama Y, Kondoh H, Takada S. Wnt proteins promote neuronal differentiation in neural stem cell culture. Biochem Biophys Res Commun. 2004; 313: 915-21.

39. Lee HY, Kleber M, Hari L, Brault V, Suter U, Taketo MM, Kemler R, Sommer $\mathrm{L}$. Instructive role of $\mathrm{Wnt} /$ beta-catenin in sensory fate specification in neural crest stem cells. Science. 2004; 303: 1020-3.

40. Yoshikawa S, McKinnon RD, Kokel M, Thomas JB. Wnt-mediated axon guidance via the Drosophila Derailed receptor. Nature. 2003; 422: 583-8.

41. Hall AC, Lucas FR, Salinas PC. Axonal remodeling and synaptic differentiation in the cerebellum is regulated by WNT-7a signaling. Cell. 2000; 100: 525-35.

42. Yu X, Malenka RC. Beta-catenin is critical for dendritic morphogenesis. Nat Neurosci. 2003; 6:1169-77.

43. Gordon MD, Nusse R. Wnt signaling: multiple pathways, multiple receptors, and multiple transcription factors. J Biol Chem. 2006; 281: 22429-33.

44. Castelo-Branco G, Arenas E. Function of Wnts in dopaminergic neuron development. Neurodegen Dis. 2006; 3: 5-11.

45. Chung S, Leung A, Han BS, Chang MY, Moon JI, Kim CH, Hong S, Pruszak $\mathrm{J}$, Isacson O, Kim KS. Wnt1-lmx1a forms a novel autoregulatory loop and controls midbrain dopaminergic differentiation synergistically with the SHH-FoxA2 pathway. Cell Stem Cell. 2009; 5: 646-58.

46. Lie DC, Colamarino SA, Song HJ, De'sire' L, Mira H, Consiglio A, Lein ES, Jessberger S, Lansford H, Dearie AR, Gage FH. Wnt signaling regulates adult hippocampal neurogenesis. Nature. 2005; 437: 1370-5.

47. Tang M, Miyamoto Y, Huang EJ. Multiple roles of beta-catenin in controlling the neurogenic niche for midbrain dopamine neurons. Development. 2009; 136: 2027-38. 